\title{
Dermatomyositis Associated with Sarcoidosis: Two Cases
}

\author{
$\underline{\text { Paschalis Sidiras }}^{1}$, Frédéric Vandergheynst ${ }^{2}$, Laurine Verset ${ }^{3}$, Hazim Kadhim ${ }^{4}$, Muhammad Shahnawaz Soyfoo ${ }^{1}$ \\ ${ }^{1}$ Department of Rheumatology and Physical Medicine, Hôpital Erasme, Université Libre de Bruxelles, Brussels, Belgium \\ ${ }^{2}$ Department of Internal Medicine, Hôpital Erasme, Université Libre de Bruxelles, Brussels, Belgium \\ ${ }^{3}$ Department of Anatomic Pathology, Hôpital Erasme, Université Libre de Bruxelles, Brussels, Belgium \\ ${ }^{4}$ Neuropathology Unit, Department of Anatomic Pathology, CHU-Brugmann, Université Libre de Bruxelles, Brussels, Belgium
}

\section{Doi: 10.12890/2016_000500- European Journal of Case Reports in Internal Medicine - @ EFIM 2016}

\begin{abstract}
Received: $12 / 09 / 2016$
Accepted: $15 / 10 / 2016$

Published: 02/11/2016
\end{abstract}

How to cite this article: Sidiras P, Vandergheynst F, Verset L, Kadhim H, Soyfoo MS. Dermatomyositis associated with sarcoidosis: two cases. EJCRIM 2016;3: doi:10.12890/2016_000500.

Conflicts of Interests: The Authors declare that there are no competing interests.

This article is licensed under a Commons Attribution Non-Commercial 4.0 License

\section{ABSTRACT}

Dermatomyositis (DM) and sarcoidosis are two idiopathic systemic disorders. Reports of patients with both conditions are extremely rare. Here we describe two patients who presented with DM and DM-associated antibodies, and later developed biopsy-proven sarcoidosis. There are increasing reports of the occurrence of sarcoidosis in the context of autoimmune diseases. These observations might imply similarities in the pathogenetic mechanisms.

\section{LEARNING POINTS}

- Sarcoidosis should be considered in patients with dermatomyositis (DM) presenting with enlarged lymph nodes. Contrary to the principle of Occam's razor, in this case one diagnosis does not rule out the other.

- The pathophysiology of sarcoidosis and DM involves both Th1 and Th17 inflammatory responses, which may explain the overlap of these two diseases

\section{KEYWORDS}

Sarcoidosis, dermatomyositis, auto-antibodies

\section{INTRODUCTION}

Dermatomyositis (DM) and sarcoidosis are two idiopathic systemic disorders. Reports of patients with both conditions are very rare, and mostly concern patients of Asian origin. Here we describe two patients presenting with DM relapsing as sarcoidosis.

\section{CASE REPORT}

Patient 1 was a 53-year-old North African woman with a history of in situ ductal breast carcinoma that was treated surgically 10 years before she presented with a violaceous rash on the dorsal aspects of her hands. She had no complaints of muscle weakness or exertional dyspnoea. Cardiopulmonary auscultation and evaluation of muscle force were normal. She had a painful periungual erythema on the 
dorsal surfaces of her distal interphalangeal (DIP), proximal interphalangeal (PIP) and metacarpophalangeal (MCP) joints, consistent with Gottron's papules, and splinter haemorrhages under her fingernails. There was no eyelid erythema. Laboratory examinations showed normal erythrocyte sedimentation rate (ESR) and C-reactive protein (CRP) levels, normal renal function, and normal electrolyte and muscle enzyme levels. A positive anti-nuclear factor was detected at a 1:5,000 titre, with identification of anti-Mi2 antibodies. The diagnosis of amyopathic DM was established, and first-line treatment with hydroxychloroquine $400 \mathrm{mg}$ daily was initiated. A computed tomography scan of the chest for cancer screening revealed nodular pulmonary lesions and bilaterally enlarged hilar mediastinal lymph nodes. Lymph node biopsy by mediastinoscopy demonstrated non-caseating granulomas (Fig. 1), without evidence of neoplasia or mycobacterial infection. Other laboratory findings were elevated angiotensin converting enzyme (ACE) (59 IU/l; normal (N)<55 IU/I) and CRP (81 mg/l; N<10 mg/l) levels. A diagnosis of sarcoidosis complicating DM was made. Follow-up 3 years later showed muscular weakness and mildly elevated creatinine phosphokinase (CK) titres, along with elevated ACE. Electromyography showed a myopathic pattern compatible with myositis, and a muscle biopsy showed necrotic muscle fibres, mostly invaded by macrophages and reactive for acid phosphatase. These fibres were mainly peripherally located and showed focal peri-fascicular atrophy (Fig. 2D). The overall picture suggested full-blown DM complicated by pulmonary sarcoidosis. Treatment with corticosteroids resulted in total remission of the inflammatory conditions.
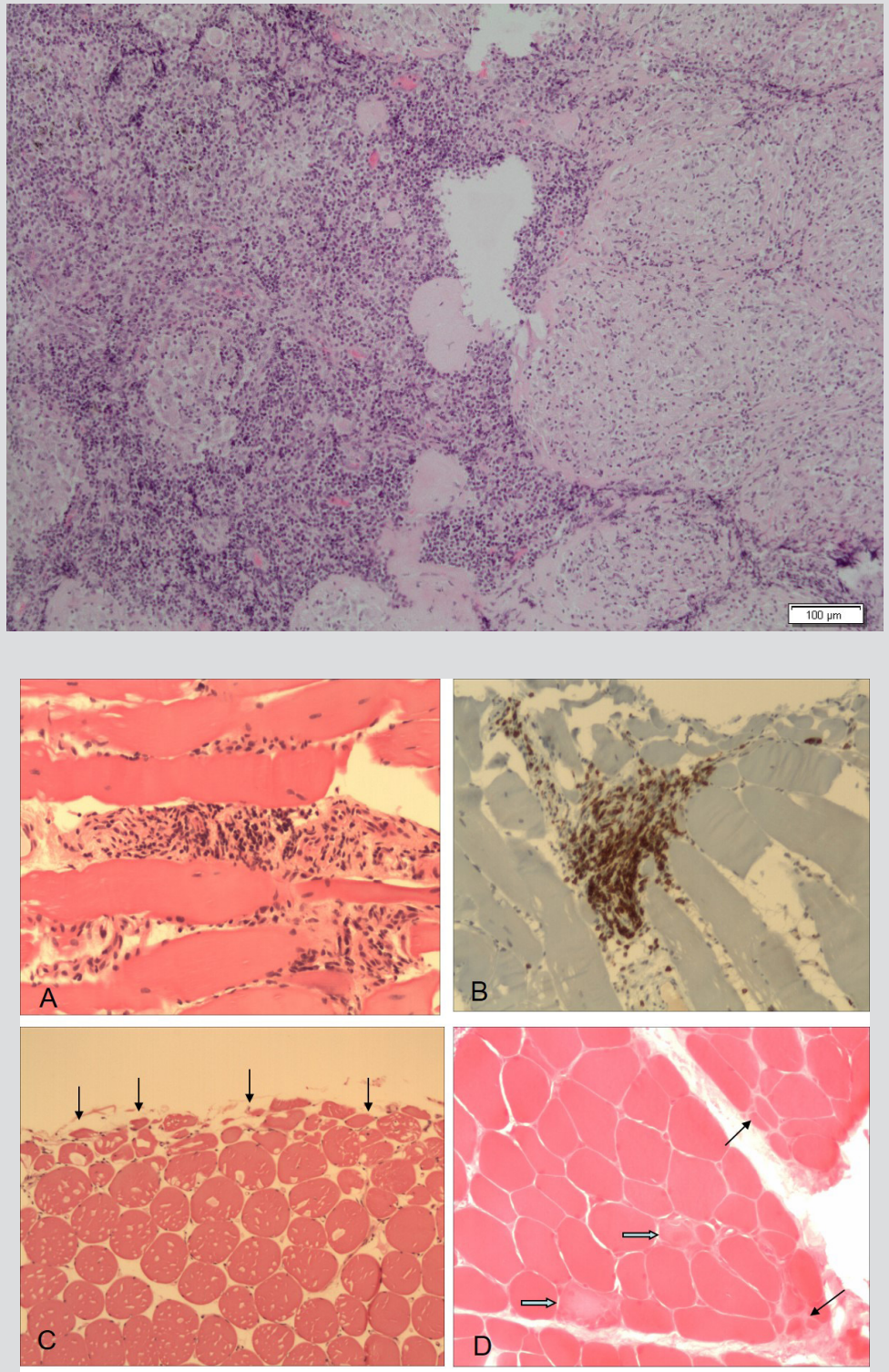
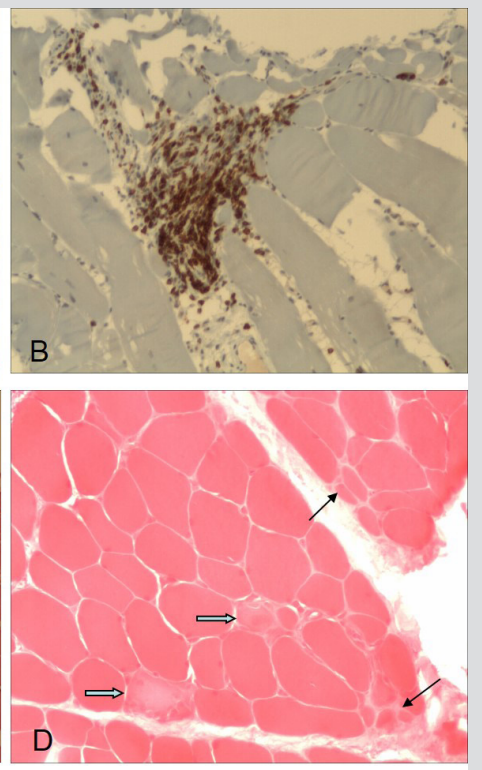

Figure 1.Lymph node biopsy findings in patient 1. Haematoxylin-eosin staining shows non-necrotizing granulomatosis of a right latero-tracheal lymph node.

Figure 2. Muscle biopsy findings. (A) Haematoxylin-eosin (H\&E) staining showing myositis characterized by necrotic fibres and particularly a marked interstitial (endomysial) inflammatory infiltrate composed mainly of mononuclear leucocytes. (B) Inflammatory cells were strongly labelled with immunohistochemical staining for CD3 (a lymphocytic marker; darkly labelled cells). (C) Horizontal sections showing perifascicular atrophy (small atrophic fibres at the periphery of fascicles; dark arrows). (D) Necrotic fibres (white arrows) invaded by macrophages and focal perifascicular atrophy (dark arrows). A,B,C: paraffin sections from patient 2; D: frozen section ( $H \& E$ stain) from patient 1. Original magnification: $\times 160(A, D), \times 100(B)$ and $\times 80(C)$. the late phase of examination. 


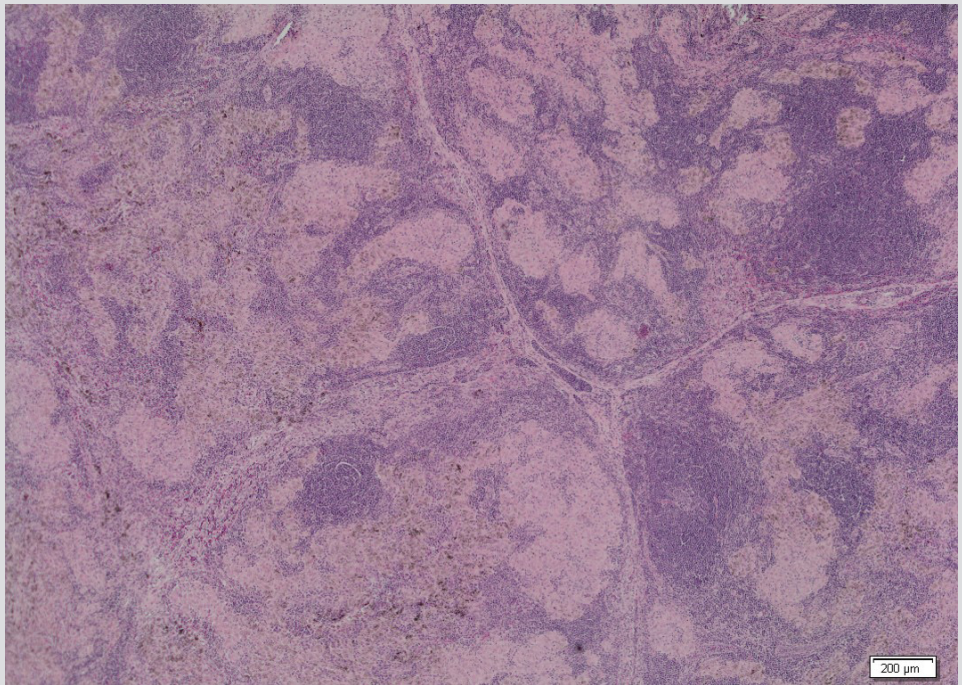

Figure 3. Lymph node biopsy findings in patient 2. Haematoxylin-eosin staining shows diffuse histocytosis of a mediastinal lymph node.

Patient 2 was a 19-year-old Caucasian woman who presented with a 2-year history of arthralgias and skin lesions that had been previously diagnosed as psoriasis. Physical examination showed Gottron's papules on both hands, while muscle examination was normal. Laboratory explorations showed normal ESR and CRP levels, markedly elevated CK levels (2800 IU/I, N<308), normal ACE levels and positive antinuclear factor at a 1:80 titre with anti-Jo1 identification. Radiographic evaluation of the lungs showed infiltrates corresponding to interstitial pneumonia. Respiratory function tests showed diminished diffusion capacity, at 68\% of DLCO/VA. Electromyography (deltoid and rectus femoris) showed a myopathic pattern suggestive of myositis. Muscle biopsy (quadriceps femoris) showed an inflammatory myositis characterized by necrotic fibres and mainly an interstitial/perivascular inflammatory mononuclear leucocytic infiltrate that was predominantly CD4-positive with immunohistological staining. There were also foci of perifascicular atrophy, and 'atypical' or 'pseudo' granulomas were sometimes suspected (Fig. $2 \mathrm{~A}-\mathrm{C}$ ). This patient with DM in a context of anti-synthetase syndrome responded well to corticosteroids. During a myositis flare 7 years later, examination showed enlarged mediastinal lymph nodes, along with an interstitial pneumonia and abnormal fixation on PET scan. Broncho-alveolar lavage showed a CD4/CD8 ratio of 0.61 ( $N>1.3)$ and elevated ACE serum levels were also observed. Lymph node biopsy revealed marked histiocytosis, compatible with the diagnosis of sarcoidosis. (Fig. 3) Immunosuppressive treatment with azathioprine and corticosteroids led to the resolution of both myositis and sarcoidosis.

\section{DISCUSSION}

We report two cases of DM complicated by sarcoidosis. We found only nine cases in the literature (mainly Asian patients) who presented with DM complicated by sarcoidosis ${ }^{[1]}$. However, there are increasing reports of the occurrence of sarcoidosis in the context of other autoimmune diseases ${ }^{[1]}$. These observations might imply a common pathogenetic pathway. Facco et al. ${ }^{[2]}$ demonstrated the role of Th1 and Th17 responses in the pathogenesis of sarcoidosis, while the marked IFN- $y$ signature in myositis and the elevated IL-17 levels underline the participation of both pathways in the development of inflammatory myopathies ${ }^{[3,4]}$. While the precise aetiology of sarcoidosis remains elusive, prolonged inflammation could be a triggering element in its pathogenesis, and chronic inflammation (including that involved in DM) might trigger the formation of granulomas ${ }^{[5]}$.

Our observations were made over a period of 7 years. As the clinical spectrum of sarcoidosis ranges from asymptomatic to life-threatening, it is possible that cases of sarcoidosis are missed in patients with other, more severe diseases. However, it remains a diagnosis that should be considered, especially since lymphadenopathy may suggest malignant disease in the setting of DM. The opposite is also valid; patients developing myositis in the context of biopsy-proven sarcoidosis could in fact be developing DM or another autoimmune disease. Myositisspecific and myositis-associated antibodies help in the diagnosis, as demonstrated by our cases.

In conclusion, we report on two cases of DM complicated by sarcoidosis, an association which is extremely rare. A common aetiopathogenic mechanism underlying these immune-inflammatory conditions is discussed. 


\section{REFERENCES}

1. Judson MA, Shapiro L, Freitas S, Polychronopoulos VS, Highland KB. Concomitant sarcoidosis and a connective tissue disease: review of the clinical findings and postulations concerning their association. Resp Med 2013;107:1453-1459.

2. Facco M, Cabrelle A, Teramo A, Olivieri V, Gnoato M, Teolato S, et al. Sarcoidosis is a Th1/Th17 multisystem disorder. Thorax 2011;66:144-150.

3. Tournadre A, Miossec P. Interleukin-17 in inflammatory myopathies. Curr Rheumatol Rep 2012;14:252-256.

4. Venalis P, Lundberg IE. Immune mechanisms in polymyositis and dermatomyositis and potential targets for therapy. Rheumatology (Oxford) 2014;53:397-405.

5. Chen ES, Moller DR. Etiologies of sarcoidosis. Clin Rev Allergy Immunol 2015;49:6-18. 